\title{
The high field magnetisation of the mixed-valence system HgSe:Fe
}

\author{
U. Zeitler ${ }^{a, *}$, S.A.J. Wiegers ${ }^{a}$, M.R.C. Overkampa , A. Wittlin ${ }^{a}$, J.C. Maan ${ }^{a}$, \\ W. Dobrowolski ${ }^{b}$, A. Mycielski ${ }^{\text {b }}$ \\ ${ }^{a}$ High Field Magnet Laboratory, University of Nijmegen, Toernooiveld 1, 6525 ED Nijmegen, The Netherlands \\ ${ }^{\mathrm{b}}$ Institute of Physics, Polish Academy of Science, 02-668 Warszawa, al. Lotnikón 32, Poland
}

\begin{abstract}
The magnetisation of the mixed-valence system $\mathrm{Hg}_{1-x} \mathrm{Fe}_{x} \mathrm{Se}$ has been measured in magnetic fields up to $20 \mathrm{~T}$. Superimposed on the monotonic background of the iron moments we observe a strong oscillatory contribution of the same order of magnitude which can be related to the de Haas-van Alphen effect of the free electrons.
\end{abstract}

\section{Introduction}

The dilute magnetic semiconductor (DMS) $\mathrm{Hg}_{1-x} \mathrm{Fe}_{x} \mathrm{Se}$ is known to reveal numerous interesting physical properties which can be related to either the free electrons or the localised moments of the iron donors [1]. For high enough doping $\left(x \geqslant 2.5 \times 10^{-4}\right.$ corresponding to an iron concentration $c \geqslant 4.5 \times 10^{18} \mathrm{~cm}^{-3}$ ) the Fermi level is pinned to a $\mathrm{Fe}^{2+}$ state $220 \mathrm{meV}$ above the conduction band edge. In this situation only a part of the iron ions act as a donor (resulting into $\mathrm{Fe}^{3+}$ ions), whereas the rest of the iron ions remain in their $2+$ valence state.

For $c>4.5 \times 10^{18} \mathrm{~cm}^{-3}$ the pinning of the Fermilevel leads to magneto-quantum oscillations of the free electron concentration and thus of the $\mathrm{Fe}^{3+}$ concentration. These oscillations can be seen by means of Hall effect measurements [2].

The magnetisation of DMSs in general, and $\mathrm{Hg}_{1-x} \mathrm{Fe}_{x}$ in particular, has recently attracted interest which mainly focused on the properties of the iron magnetic moments

*Corresponding author. in the host lattice and, in particular, on the anisotropy of the magnetic interactions [3, 4]. At low fields also de Haas van Alphen (dHvA) measurements have been performed in $\mathrm{Hg}_{1-x} \mathrm{Fe}_{x} \mathrm{Se}$ [5] and analysed in terms of a closed-orbit magnetic breakdown model.

In this work we consider the properties of the conduction electrons and their interaction with the iron donors. On the monotonic background of the magnetisation of the $\mathrm{Fe}$ ions we observe a strong oscillatory signal the main part of which can be attributed to the dHvA effect of the free electrons. We present some first experimental results and compare them to Shubnikov-de Haas $(\mathrm{SdH})$ and Hall effect data on samples which were cut from the same piece.

\section{Experimental}

The samples were cut with a band saw to rectangular pieces of about $3 \times 1.5 \times 1.5 \mathrm{~mm}^{3}$ for the magnetisation measurements and $7 \times 1.5 \times 1.5 \mathrm{~mm}^{3}$ for the transport experiments. We have studied four different samples (for each transport and magnetisation) with a nominal iron content of $2.5,5,7.5$, and $10 \times 10^{18} \mathrm{~cm}^{-3}$. 
For measuring the magnetisation of the samples we used a home-built high-precision torque magnetometer. It consists of a rotatable disk with 6 silver paint electrodes mounted on a $50 \mu \mathrm{m}$ manganin torque wire. This disk faces a fixed plate with the same number of electrodes. The sample is mounted on the rotatable disk at a distance $d$ from the axis of the torque wire. A magnetisation $\boldsymbol{M}$ of the sample will lead to a torque on the device given by $\boldsymbol{T}=\boldsymbol{\nabla}(\boldsymbol{M} \cdot \boldsymbol{B}) \times \boldsymbol{d}+\boldsymbol{M} \times \boldsymbol{B}$ which contains two contributions: a term arising from the force $M \cdot \nabla B$ on the sample reflecting the absolute value of the magnetisation and a second contribution $\boldsymbol{M} \times \boldsymbol{B}$ arising from anisotropy in the magnetization.

The two contributions to the torque can be separated by measuring in different field gradients. Specifically, in the quadratic field profile of a Bitter coil the field gradient depends linearly on the vertical distance $h$ of the torque meter from the centre of the field and is proportional to the applied magnetic field: $\nabla B=-B_{0} h / h_{0}^{2}\left(B_{0}\right.$ is the magnetic field in the center of the coil and $h_{0}=13 \mathrm{~cm}$ in the coil we used).

The torque is obtained by measuring the capacitance between the electrodes. The device is calibrated using a feedback coil mounted on the rotatable disk which produces a magnetic moment $\boldsymbol{M}$ perpendicular to the field and to the torque wire.

The sensitivity of the torque meter is about $10 \mathrm{pNm}$ which allows to measure magnetic moments of the order of some $10^{-10} \mathrm{~J} / \mathrm{T}$.

\section{Results}

In Fig. 1a we show the quantity $M^{*}:=T / B$ which can be written as

$$
M^{*}=M \cdot(\nabla B) / B d+M \times B / B
$$

as a function of the magnetic field for different values of the field gradient. $M^{*}$ is proportional to the magnetisation of the sample. The vertical distance $h$ of the torque meter from the center of the field is indicated for each curve and the corresponding field gradients are given in the figure caption.

The dependence of $M^{*}$ from position and orientation of the sample reflects the two terms of $M^{*}$ appearing in Eq. (1) namely the isotropic magnetisation sensitive to the field gradient and an anisotropic contribution $\boldsymbol{M} \times \boldsymbol{B} / \boldsymbol{B}$. In these first experiments the sample has been arbitrarily oriented. Even in a strong field gradient the main contribution to $M^{*}$ arises from the term $\boldsymbol{M} \times \boldsymbol{B}$ (which only slightly depends on position) showing the

\footnotetext{
${ }^{1} \mathrm{~A}$ more detailed description of the torque meter is given else-
} where in these proceedings [6].
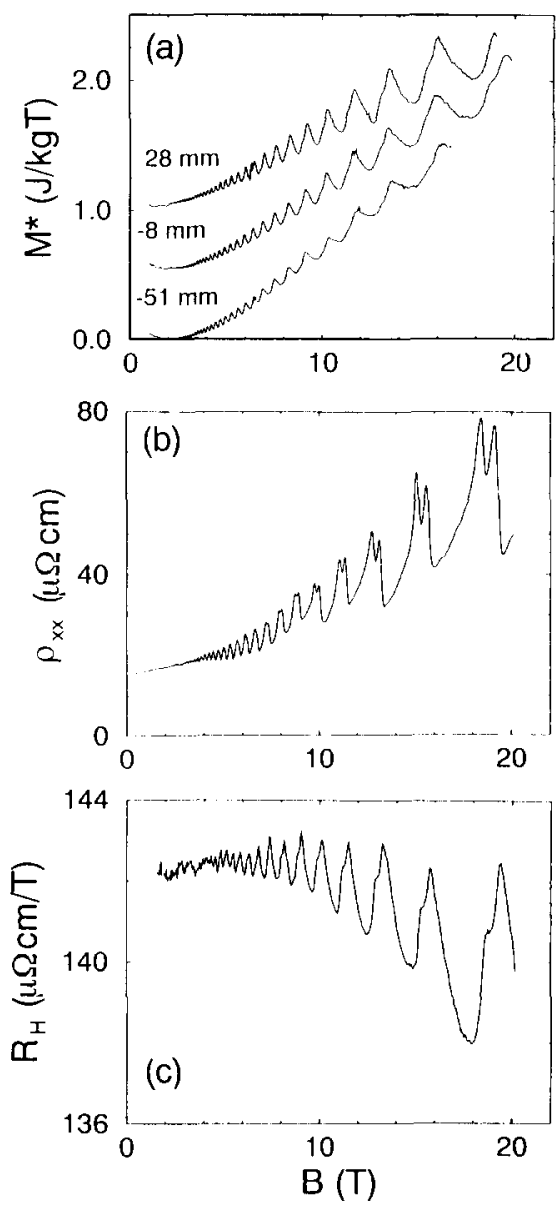

Fig. 1. Typical results for sample \#3 $\left(c \approx 7.5 \times 10^{18} \mathrm{~cm}^{-3}\right)$ at $4.2 \mathrm{~K}$. (a) The magnetic field dependence of the normalized magnetisation $M^{*}$ for different vertical positions of the torque meter (the curves are shifted for clarity). The vertical positions $h=28 \mathrm{~mm}(-8 \mathrm{~mm},-51 \mathrm{~mm})$ correspond to field gradients $\nabla B=-17 \mathrm{~T} / \mathrm{m}(5 \mathrm{~T} / \mathrm{m}, 37 \mathrm{~T} / \mathrm{m})$ at $B=10 \mathrm{~T}$. (b) The resistivity $\rho_{x x}$ and (c) the Hall constant $R_{\mathrm{H}}$.

high sensitivity of the device to an anisotropy in the magnetisation.

At a given position $M^{*}$ is composed of two contributions: a monotonic background arising from the localised moments of either $\mathrm{Fe}^{2+}$ or $\mathrm{Fe}^{3+}$ and a strong oscillatory part. Both contributions contain isotropic and anisotropic terms. The oscillations are periodic in $1 / B$ and have the same period as the $\mathrm{SdH}$ oscillations of the resistivity $\rho_{x x}$ (Fig. 1(b)). The anisotropic part in the background to $M^{*}$ reflects the anisotropy of the $\mathrm{Fe}^{2+} / \mathrm{Fe}^{3+}$ magnetisation. $^{2}$

\footnotetext{
${ }^{2}$ This part is investigated in more detail in Ref. [3]
} 
A part of the oscillatory part might originate from a magneto-quantum oscillations of the free electrons. These oscillations lead to an oscillating redistribution of the $\mathrm{Fe}^{2+} / \mathrm{Fe}^{3+}$ moments [2]. Since the $\mathrm{Fe}^{3+}$ and the $\mathrm{Fe}^{2+}$ have a different magnetic moment this also causes magneto-quantum oscillations of the iron magnetisation.

The quantum oscillations of the free electron concentration $n_{\mathrm{f}}$ can be visualized in the Hall constant $R_{\mathrm{H}}:=\rho_{x y} / B=1 / n_{\mathrm{f}} e$ as shown in Fig. 1(c). They are only of the order of a few percent and therefore cannot explain the large oscillations in the magnetisation. Furthermore, the dependence of the oscillatory part in the magnetisation on the position of the samples is different compared to the monotonic background (Fig. 1(a)).

A main part of the oscillatory contributions to the magnetisation arises not from localized iron moments but has its origin in the dHvA effect of the conduction electrons. Also this contribution contains a dominating anisotropic part which can be related to the anisotropy of the Fermi surface [5] and to the influence of the sample geometry on the direction of the macroscopic magnetisation.

Finally, it might be interesting to remark a main difference between the SdH effect and the $\mathrm{dHvA}$ effect as visible in these data (Figs. 1(a) and (b)). The spin splitting is clearly resolved in the first but merely visible in the latter. This can be crudely understood using the fact that higher-order terms in the $\mathrm{dHvA}$ oscillations are strongly damped compared to SdH oscillations [7]. A further quantitative comparison of SdH and dHvA effect might yield important information about the electronic properties of a quasi-free three-dimensional electron gas in quantising magnetic fields.

\section{Conclusions}

We have presented some first results on the oscillatory high field magnetisation of iron doped HgSe. They can be attributed to the dHvA effect of the free electrons. Further investigations concerning the dependence of $\mathrm{dHvA}$ oscillations as well as of the magnetisation of the localised iron moments on orientation will follow. It would also be of great interest to use these highly doped semiconductors for a quantitative comparison between the dHvA effect and the SdH effect in a well defined system where both of them occur.

\section{References}

[1] See e.g. A. Twardowski, in: Diluted Magentic Semiconductors, ed. M. Jain (World Scientific Publishing, Singapore, 1991).

[2] M. von Ortenberg, O. Portugall, W. Dobrowolski, A. Mycielsky, R. Galazka and F. Herlach, J. Phys. C. 21 (1988) 5393; I. Laue, O. Portugall, M. von Ortenberg and W. Dobrowolski, in: High Magnetic Fields in Semiconductor Physics III, Springer Series in Solid State Physics, Vol. 101, ed. G. Landwehr (Springer, Berlin, 1992).

[3] K. Dybko, A. Lusakowski, J. Kossut, M Arciszeweska, P.J.E.M. van der Linden, A. Wittlin, J.A.A.J. Perenboom and A. Mycielski, in: Proc. XXIII Internat. School on Physics of Semiconducting Compounds (Jasowiec, Poland, 1994).

[4] Z. Wilamowski, H. Przybylinska, W. Joss and M. Guillot. Semicond. Sci. Technol. 8 (1993) S44.

[5] M.M. Miller and R. Reifenberger, Phys. Rev. B 38 (1988) $3423,4120$.

[6] S.A.J. Wiegers, J.C. Maan and C.T. Foxon, Physica B 211 (1995) 474.

[7] See, for example, L.M. Roth and P.N. Argyres, in: Physics of III-V Compounds, ed. O. Madelung (Wiley, New York, 1964). 